\title{
Potencial da Aplicação de Blockchain para a Rastreabilidade de Cadeias de Alimentos Sustentáveis: um estudo prospectivo
}

\author{
Potential of Blockchain Application for Sustainable Food Chain \\ Traceability: a prospective study
}

\author{
Andrik Guimarães Ferreira ${ }^{1}$ \\ Rosinei de Sousa Oliveira ${ }^{1}$ \\ Cristina M. Quintella ${ }^{2}$ \\ Celson Pantoja Lima ${ }^{1}$ \\ ${ }^{1}$ Universidade Federal do Oeste do Pará, Santarém, PA, Brasil \\ ${ }^{2}$ Universidade Federal da Bahia, Salvador, BA, Brasil
}

\begin{abstract}
Resumo
O futuro sustentável, segundo a ONU, faz referência às ações que garantam a sustentabilidade dos sistemas produtivos perante os recursos naturais. Os desafios aqui destacados são de busca pelo equilibrio entre a expansão da produção $e$ a fraude alimentar. Este estudo tem por objetivo realizar uma análise prospectiva em patentes e em publicações científicas para fornecer uma visão geral a respeito do desenvolvimento de soluções tecnológicas sobre blockchain aplicado à rastreabilidade em cadeias de suprimentos sustentáveis. A partir da análise dos documentos das bases de dados Scopus e de patentes pelo software Questel Orbit, observa-se que a China e os Estados Unidos se destacam por definirem estratégias de política nacional, tanto do ponto de vista comercial em proteção de patentes como de pesquisas científicas. Porém, apesar do grande espectro de aplicação da tecnologia, o estudo demonstra que ainda há carência de soluções que relacionem a rastreabilidade de cadeias com o contexto de sustentabilidade.
\end{abstract}

Palavras-chave: Blockchain. Cadeia de Suprimentos. Sustentabilidade.

\begin{abstract}
The sustainable future, according to the $\mathrm{UN}$, refers to actions that guarantee the sustainability of the productive systems included in natural resources. The challenges highlighted here are the search for a balance between expanding production and food fraud. This study aims to carry out a prospective analysis on patents and scientific publications to provide an overview regarding the development of technological solutions on blockchain applied to traceability in sustainable supply chains. From the analysis of the documents in the Scopus and patent databases by the Questel Orbit software, it is observed that China and the United States stand out for defining national policy strategies, both from the commercial point of view of patent protection and research scientific. However, despite the wide spectrum of application of the technology, the study shows that there is still a lack of solutions that relate the traceability of chains with the context of sustainability.
\end{abstract}

Keywords: Blockchain. Supply Chain. Sustainability.

Área Tecnológica: Prospecção Tecnológica. Inteligência Artificial. 


\section{Introdução}

A Agenda 2030 da ONU para o Desenvolvimento Sustentável, em seu conjunto de objetivos e metas, propõe dobrar a produtividade agrícola, garantir sistemas sustentáveis de produção de alimentos e, ainda, manter e preservar os ecossistemas. Esse novo panorama apresenta dois desafios, por isso, considera-se aqui pertinente evidenciá-los pois eles apresentam relativa aderência com o escopo deste trabalho. O primeiro está relacionado com o obstáculo em estabelecer um equilíbrio entre a expansão da produção agrícola e o uso racional da terra. Enquanto o segundo envolve a urgência no desenvolvimento de mecanismos que evitem fraude alimentar dentro de um cenário em que seja possível, por exemplo, ter conhecimento de todos os processos envolvidos na cadeia de produção de um determinado alimento.

Hoje, o setor de produção agrícola está diante de um grande paradoxo. Se, por um lado, é evidente a crescente demanda pela produção de alimentos, por outro, é mandatório reduzir os impactos ambientais em virtude de um esforço para fortalecer a resposta global à ameaça das mudanças climáticas, tendo como um dos fatores principais o desmatamento (RYAN, 2019). O futuro sustentável busca mecanismos garantidores de sustentabilidade dos sistemas produtivos perante os recursos naturais e, para que esse objetivo seja alcançado, são necessárias ações público-privadas, financiamentos e investidores.

Com o passar dos anos fica cada vez mais evidente que o desmatamento não gera desenvolvimento, pelo contrário, inibe investimentos. Esse entendimento leva o nível do debate para um outro patamar, uma vez que grandes projetos no setor agrícola são dependentes, na maioria dos casos, de investimentos privados internacionais. Percebe-se que o esforço sustentável tende a seguir a lógica de um discurso com efeito prático, inclusive ressalta-se que uma das maiores produtoras de salmão do mundo suspendeu a compra de ração de base proveniente da soja de uma trade que atua na região amazônica por consequência da não garantia de políticas voltadas para a diminuição dos impactos ambientais (BYRNE, 2020).

Esse contexto também tem relação com o segundo desafio, o de fraude alimentar, que implica obrigatoriedade de informações quanto à origem da produção. Os consumidores estão cada vez mais exigindo transparência de toda a cadeia dos alimentos por eles consumidos - a real origem, o efeito de seus métodos de produção em nosso planeta e como se dá a relação dos animais no processo de produção (IBM, 2018). Ademais, uma pesquisa realizada com 1.500 pessoas nos Estados Unidos indicou que "[...] 70\% dos consumidores apontaram que querem varejistas mais transparentes sobre seus esforços de sustentabilidade" (HARTMAN GROUP, 2017). Tal como a postura da Walmart, maior varejista norte-americana, que vem exigindo desde de 2015 da Las Palmas, empresa da Guatemala produtora de óleo de palma, um certificado de sustentabilidade para continuar fornecendo matéria-prima à estadunidense (ABADI; BALL, 2020).

Nesse cenário, companhias investem na adoção de tecnologias que se mostram capazes de serem utilizadas para reduzir gaps de (des)informação no âmbito sustentável e da segurança alimentar. Uma dessas tecnologias é denominada de blockchain, em maioria, utilizada por players do mercado financeiro e criptomoedas (LESSAK; DIAS; FREY, 2018). A compreensão dessa tecnologia não é intuitiva, e a exploração de todos os seus conceitos fogem do escopo deste trabalho. Porém, de forma resumida, o bloco que contém as informações é replicado nos milhares de outros blocos que compõem a cadeia, garantindo a imutabilidade das informações 
inseridas. Por exemplo, uma possível adulteração em um registro que contém a informação de origem de um alimento implicaria na edição das informações em todos os blocos (i.e. armazenado em computadores diferentes) da cadeia. Do ponto de vista da capacidade computacional que se tem hoje, a adulteração sem a permissão dos outros blocos é impossível.

Nas cadeias de suprimentos, as informações digitais de um produto, como detalhes da origem da fazenda, números de lotes, temperaturas de armazenamento e detalhes do transporte, podem ser conectadas digitalmente aos itens alimentares, e as informações são inseridas no blockchain a cada etapa do processo (RAMANURTHY, 2016). A IBM, por exemplo, importante player do mercado de tecnologia, já oferece blockchain no seu portfólio e criou o Food Trust, uma solução para o rastreio de cadeias de suprimentos de alimentos que já é utilizada por grandes varejistas como Walmart, Dole e Nestlé (ANZALONE, 2020). Outros diversos exercícios de planejamento têm sido usados para orientar a reflexão e a elaboração de estratégias para essa nova tecnologia. Porém, boa parte dessas soluções está sendo utilizada para rastreabilidade de cadeias de suprimentos tradicionais.

Desse modo, este trabalho faz uso da metodologia de prospecção tecnológica em patentes e em publicações científicas com o objetivo de fornecer uma visão geral a respeito do desenvolvimento de soluções baseadas em blockchain aplicado à rastreabilidade em cadeias de suprimentos de alimentos no mundo. Não obstante, até o desenvolvimento deste trabalho não foram encontradas aplicações do uso do blockchain como ferramenta que relacione a rastreabilidade de cadeias de suprimentos como garantia de origem de sustentabilidade e de monitorização na região amazônica, interesse também de estudo para os autores.

Na Amazônia há importantes cadeias que podem se beneficiar desse tipo de solução. Uma delas é a cadeia de palma na qual o Estado do Pará concentra cerca de $85 \%$ da produção de dendê nacional, fazendo com que o Brasil ocupe o $5^{\circ}$ lugar na produção mundial (ABRAPALMA, 2018). Dessa forma, a rastreabilidade da cadeia com blockchain e a possibilidade de diminuir os intermediários na cadeia se apresentam como propostas promissoras para alterar o modelo de negócio que se estende por anos no segmento. Isso, além de representar um ganho econômico limitado, ainda não impede que os pequenos agricultores desenvolvam a produção de forma eficiente e sustentável.

A elaboração deste estudo é parte de uma pesquisa mais ampla que tem por objetivo desenvolver um roadmap tecnológico do uso de blockchain aplicado à cadeia produtiva do óleo de palma e os potenciais cenários no contexto da Amazônia. O tema também está inserido dentro do escopo do projeto Desenvolvimento Sustentável de Processos e Produtos na Amazônia que visa à integração entre programas de pós-graduação de universidades federais do Pará e da Bahia para atender à necessidade de desenvolvimento sustentável inerentes às regiões envolvidas.

\section{Metodologia}

Este trabalho se concentra, nas duas primeiras etapas, de um total de cinco, na metodologia de roadmap tecnológico proposto por Borschiver e Silva (2016), ilustrada na Figura 1 (A). A primeira etapa, "Etapa Pré-Prospectiva", é realizada por meio de uma pesquisa preliminar e geral relacionada ao tema do estudo. Optou-se por utilizar o Google ${ }^{\circledR}$ com os termos relacionados aos tópicos de "Blockchain", "Cadeias de Suprimentos", "Rastreabilidade" e "Sustentabilidade". 
Ainda na etapa Pré-Prospectiva identificou-se a ferramenta Strategic Intelligence do World Economic Forum (2020) que classifica as aplicações do blockchain em áreas, conforme é ilustrado na Figura 1 (B). A partir disso, os escopos das etapas posteriores passaram a ser especificados de acordo com os temas dispostos nas áreas "Futuro do Meio Ambiente e Segurança de Recursos Naturais (i.e. fatia realçada de cor vermelha), e Cadeias de Suprimentos e Transporte (i.e. fatia realçada de cor laranja). Como o objetivo dessa fase pretende, além de identificar a familiaridade, definir a estratégia de pesquisa, decidiu-se por aprofundar a busca com relação ao que se dispõe de tecnologias e de pesquisas em andamento.

Figura 1 - (A) Metodologia desenvolvimento das etapas de prospecção; (B) Áreas e subáreas de estudo de blockchain do World Economic Forum

(A)

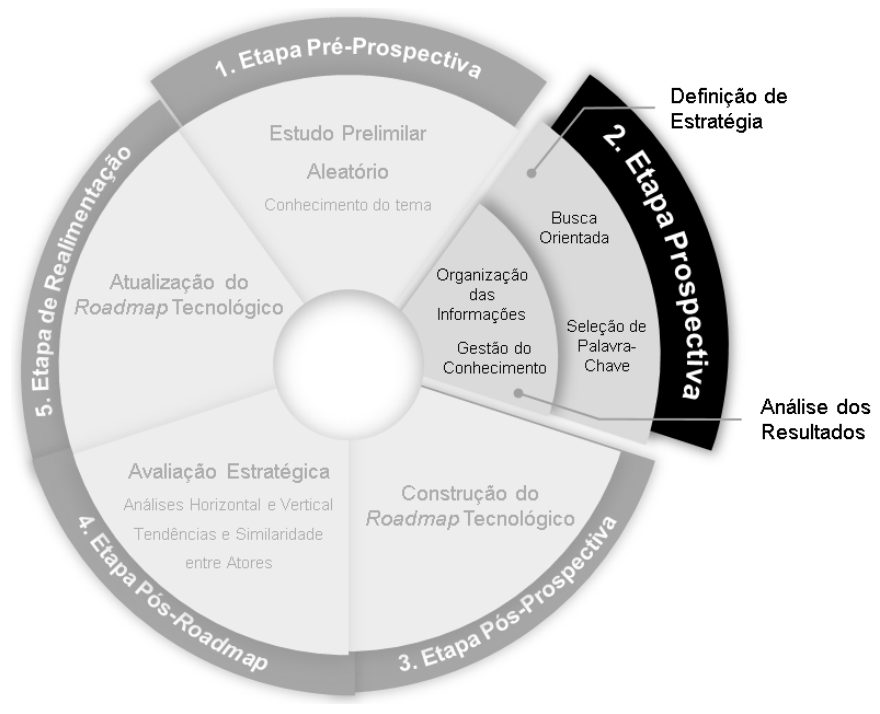

(B)

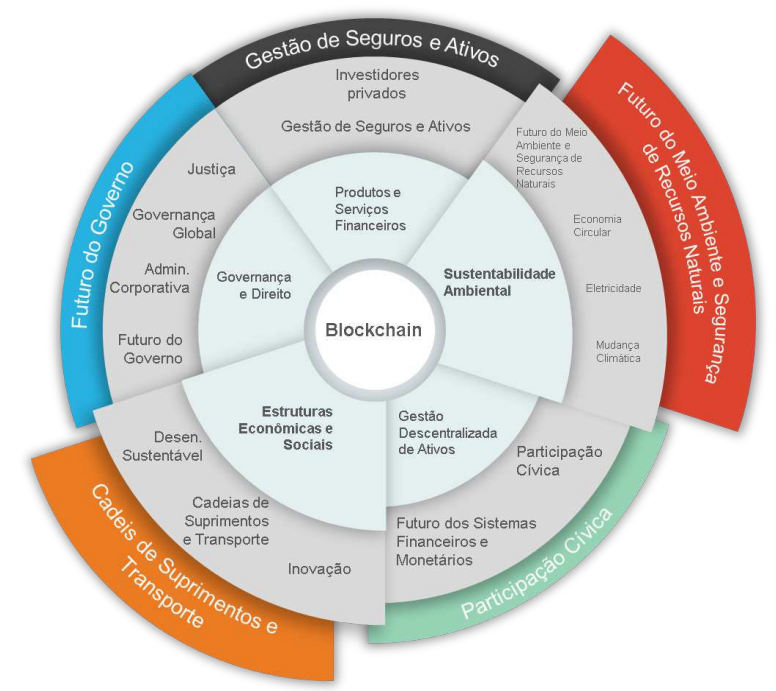

Fonte: (A) Adaptada de Borschiver e Silva (2016); (B) Elaborada pelos autores a partir da ferramenta Strategic Intelligence do World Economic Forum (2020)

A "Etapa Prospectiva", segunda etapa, é composta de busca direcionada de informações em bases de dados especializadas, assim como a análise desses dados e sua transformação em conhecimento (BORSCHIVER; SILVA, 2016). O trabalho recorre às publicações científicas 
utilizando a base de dados Scopus, que possui a maior base de dados de resumos e de citações de literatura revisada por pares; com ferramentas para analisar e visualizar a pesquisa, número superior a 500 anais de conferências onde se tem presente mais de 70 sociedades importantes, como IEEE, ACM, IEE, SPIE (ELSEVIER, 2020); patentes depositadas e concedidas (CQUESTEL ORBIT, 2020), de 2011 a 2020; e base de dados do Instituto Nacional da Propriedade Industrial (INPI), seção Programa de Computador. O objetivo de utilizar essas bases é identificar tendências tecnológicas e mercadológicas do uso de blockchain nas cadeias de suprimentos sustentáveis quanto ao rastreamento.

\subsection{Estratégias de Buscas I e II para Patentes e Publicações Científicas}

Por meio da Prospecção Tecnológica é possível identificar o estágio de maturidade da tecnologia que é buscada e como ela se insere na sociedade (QUINTELLA et al., 2011). Com isso, foram definidos dois escopos de buscas, a Estratégia de Busca I apoiou-se em palavras-chave e na Classificação Internacional de Patentes (CIP). Com intuito de evitar que a pesquisa apresentasse uma discrepância em razão de outros termos que fizessem referência a blockchain, pelo fato de ser um termo relativamente novo, e para aprimorar as buscas na tentativa de englobar referências passadas, foram elencados termos sinônimos dessa tecnologia, que são estes: "cryptographic ledger" e "distributed ledger". A partir disso, a Estratégia de Busca II fez uso de palavras-chave como IPC e seus sinônimos.

Adotou-se para buscas dos documentos as seguintes palavras-chave: "Blockchain", "Supply Chain", "Traceability", "Sustainable", ademais, foram realizadas combinações aplicadas em conjunto dos operadores booleanos AND, OR, às palavras-chave escolhidas anteriormente, formando os strings de busca para base patentária e de publicações científicas, exemplificadas no Quadro 1.

Nos resultados das buscas, foram destacados o ano de publicação para documentos científicos e o ano de publicação das patentes; o país de origem do autor e do depositante da patente; o tipo de autor ou depositante (universidades, centros de pesquisa ou empresas); $e$ outras informações.

Esta pesquisa, com dados buscados nas bases de dados indicados, ocorreu de abril a junho de 2020. A seguir estão sumarizadas as strings de buscas utilizadas na pesquisa em bases de dados de patentes, com uso da suíte Orbit Intelligence, e também as strings de buscas de publicações científicas na base de dados da Scopus.

Quadro 1 - Metodologias utilizadas para pesquisas de patentes no Questel Orbit e Scopus

\begin{tabular}{|c|c|}
\hline String Metodologia de Busca I (PATENTES) & String MetOdologia De BusCa II (PATENTES) \\
\hline \begin{tabular}{c|c} 
(BLOCK CHAIN) AND (SUPPLY CHAIN \\
OR TRACEABILITY OR SUSTAINABLE)
\end{tabular} & $\begin{array}{c}\text { (BLOCK CHAIN OR DISTRIBUTED LEDGER } \\
\text { OR CRYPTOGRAPHIC LEDGER) AND (SUPPLY } \\
\text { CHAIN OR TRACEABILITY OR SUSTAINABLE) }\end{array}$ \\
\hline String de busca para Scopus \\
\hline (BLOCKCHAIN AND "SUPPLY CHAIN" OR "TRACEABILITY" OR "SUSTAINABLE")
\end{tabular}

Fonte: Elaborado pelos autores deste artigo 


\section{Resultados e Discussão}

A partir da definição dos escopos de buscas e das bases de dados escolhidas, esta seção trata dos resultados recuperados tanto a quantidade como a discussão dos pontos mais relevantes do estudo. A partir daqui, estão subdivididos para melhor compreensão em dois itens: (3.1) Patentes e (3.2) Publicações Científicas.

\subsection{Patentes}

No escopo 1, com a concatenação, recuperou-se 882 famílias de patentes, dos quais 762 são patentes solicitadas (pending), 49 são patentes concedidas (granted) e 11 estão revogadas ou mortas (dead). Já na utilização de escopo 2, recuperou-se 895 famílias de patentes, sendo 32 solicitadas (pending), 50 concedidas (granted) e 13 revogadas ou mortas (dead).

No Gráfico 1 é possível verificar que, entre as classificações mais utilizadas para patentes por área, a seção "G" apresenta maior quantidade e faz referência à área de Física, seguida pela seção "H", que indica a área de Eletricidade. Para melhor refinamento e exemplificação, foram excluídas as subclasses que não fazem referência ao objetivo desta pesquisa (como exemplo, destaca-se a área especificamente de serviços bancários), resultando em cinco, que estão destacados pelas cores verde e vermelho.

Gráfico 1 - (A) Família de patentes por IPC; (B) Família de patentes por IPC, mais sinônimos

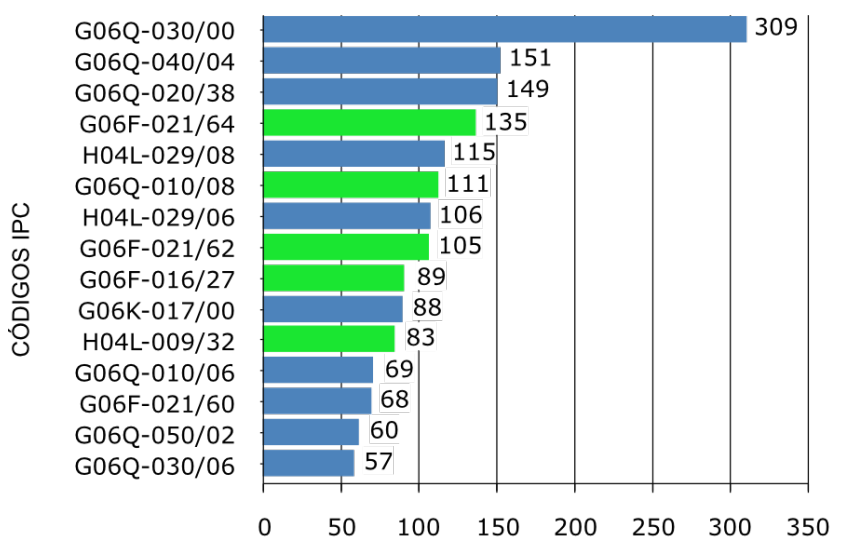

$\mathrm{B}$

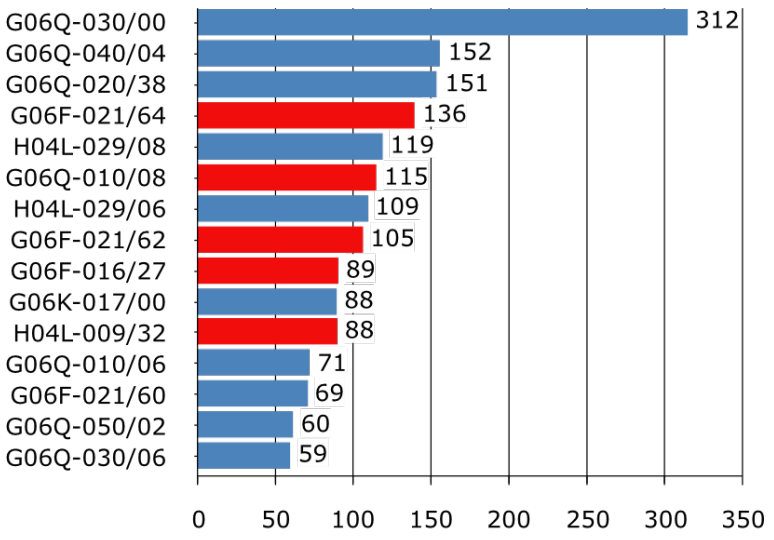

NÚMERO DE FAMÍLIAS DE PATENTES

Fonte: Elaborado pelos autores deste artigo a partir da base de dados Orbit Intelligence (2020)

Das subclasses em destaque pelos escopos de buscas analisadas segundo a CIP, nota-se que ainda não há classificação exata para tecnologia blockchain. Porém, ao solicitar depósitos, é possível observar uma tendência para alguns códigos, cujas descrições se referem a: G06F-021/64 (Protegendo a integridade dos dados, por exemplo, usando somas de verificação, certificados ou assinaturas), com 135 famílias de patentes; G06Q-010/08 (Logística, por exemplo, entreposto, carregamento, distribuição ou expedição; Gerenciamento de estoque ou estoque, por exemplo, preenchimento, suprimento ou equilíbrio de pedidos) que possui 111 famílias de patentes.

Ainda da seção "G" tem G06F-021/62 (Protegendo o acesso aos dados por meio de uma plataforma, por exemplo, usando chaves ou regras de controle de acesso) e G06F-016/27 
(Replicação, distribuição ou sincronização de dados entre bancos de dados ou dentro de um sistema de banco de dados distribuído; Arquiteturas de sistema de banco de dados distribuído para isso) com 105 e 89 famílias de patentes, respectivamente. Na seção "H" encontra-se a subclasse H04L-009/32 (Incluindo meios para verificar a identidade ou autoridade de um usuário do sistema), tendo 83 famílias de patentes.

Após equalização dos códigos patentários, foram refeitas as buscas com adição dos cinco códigos destacados no gráfico anterior. Os novos resultados são observados a partir do Gráfico 2, obteve-se 411 famílias de patentes, das 882 inicialmente recuperadas, e a primeira publicação de patente foi realizada na área de blockchain aplicado a cadeias de suprimentos para rastreabilidade no ano de 2014. Ao realizar a busca com escopo 2 , alterou-se apenas a quantidade de patentes, tendo sido recuperadas 423 famílias de patentes, visto que a data das primeiras publicações é igual.

O ano de 2014 foi marcado por diversos escândalos sobre procedência de alimentos. Vários fornecedores chineses foram investigados pela entrega de produtos como carne e óleo vegetal de origem duvidosa. Aliado a isso, funcionários de uma subsidiaria da Walmart na China denunciaram fraude alimentar de produtos, o escândalo afetou a reputação do Walmart no mercado de alimentos chinês, avaliado em 1 trilhão de dólares (HE, 2014; PETERSON, 2014).

Com base na Estratégia de Busca I, no biênio 2016 e 2017, houve um aumento tímido nos números de publicações, e, em 2018 e 2019, o "ápice", que somado, é de 331 publicações. Até a data desta pesquisa, foram recuperadas apenas 42 publicações em 2020, esse número logicamente deverá aumentar no decorrer do ano, no mais, ele pode sugerir que existe instabilidade no desenvolvimento de tecnologias que visam a aperfeiçoar a rastreabilidade em blockchain aplicado a cadeias de suprimentos.

Gráfico 2 - (A) Número de patentes publicadas por ano; (B) Número de patentes com sinônimos publicadas por ano

A

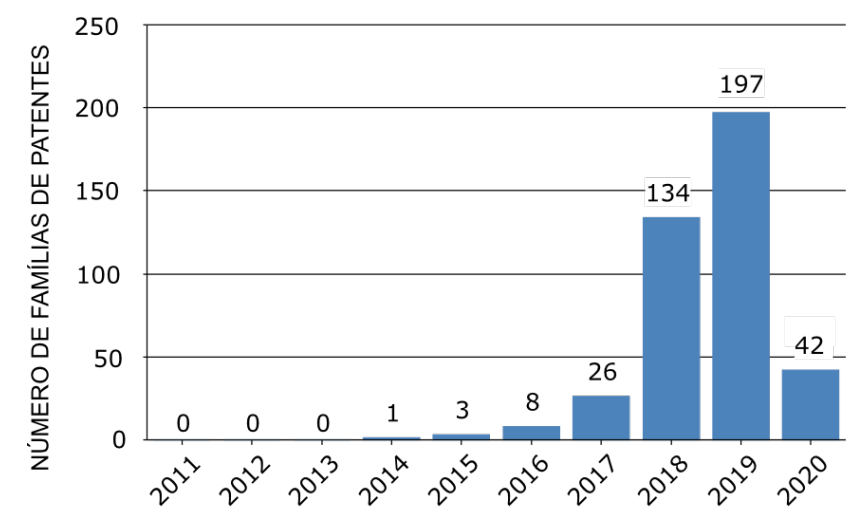

B

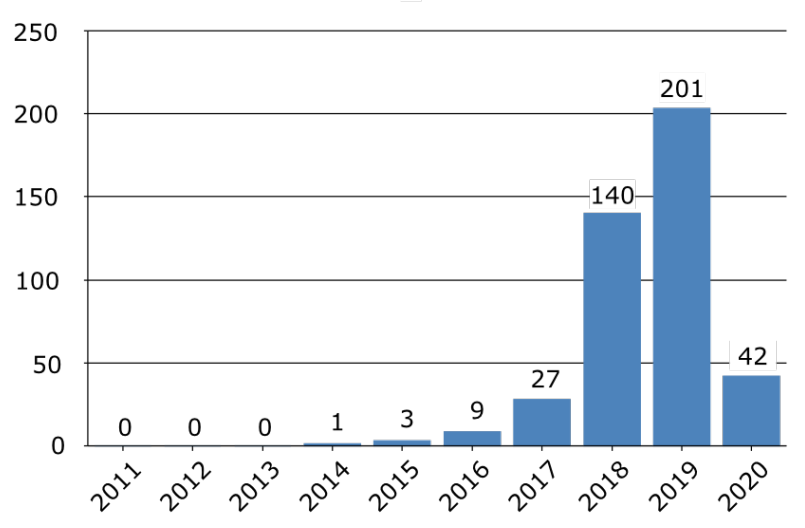

FAMÍLIAS DE PATENTES POR ANO DE PUBLICAÇÃO

Fonte: Elaborado pelos autores deste artigo a partir da base de dados Orbit Intelligence (2020)

O Gráfico 3 clarifica o cenário de proteção de patentes no mundo, mostra quais países protegem patentes relacionadas com blockchain, cadeias de suprimentos e rastreabilidade. Dentro da Estratégia de Busca I, é possível visualizar que a China (CN) possui 338 depósitos, sendo esse número mais que o dobro das patentes protegidas dos outros país somados, o que 
demonstra que a China está à frente de patentes quando se trata de quantidade, seguida dos Estados Unidos (US), com 49 patentes no setor, e a Organização Europeia de Patentes (EP) em terceiro, com 33 patentes. Na Estratégia de Busca II, a variação é vista apenas na quantidade de número de depósitos. A indústria chinesa já se movia rapidamente em torno da tecnologia blockchain pelo potencial de aplicabilidade e, agora, ainda mais, após o endosso do governo para o desenvolvimento de blockchain como uma prioridade nacional (AN, 2019); esse esforço alia-se à simplificação do processo para pedido de patentes blockchain pela Administração Nacional de Propriedade Intelectual da China (NIPA), o país tende a se manter líder de patentes no mundo (CANT, 2020).

Com relação ao Brasil (BR), é possível visualizar duas patentes protegidas: na primeira, o depositante é a empresa francesa BULL e refere-se ao código IPC G06F-021/64 (BULL, 2019); o segundo depositante é Marco Tulio de Oliveira Raposo, cuja invenção depositada descreve um equipamento de rastreabilidade para cadeia de leite e derivados, a tecnologia tem como referência o código IPC G06Q-010/08 (RAPOSO, 2020). Dentro das análises de códigos patentários (Gráfico 1), os dois depósitos citados estão nas classificações com maior número de patentes recuperadas (seção $G$ ).

Gráfico 3 - (A) Número de patentes por países de proteção; (B) Número de patentes com sinônimos por países de proteção

A

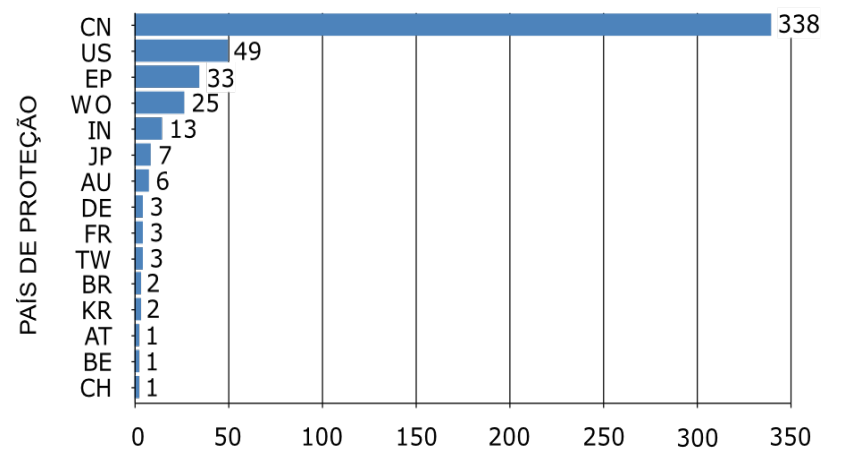

$\mathrm{B}$

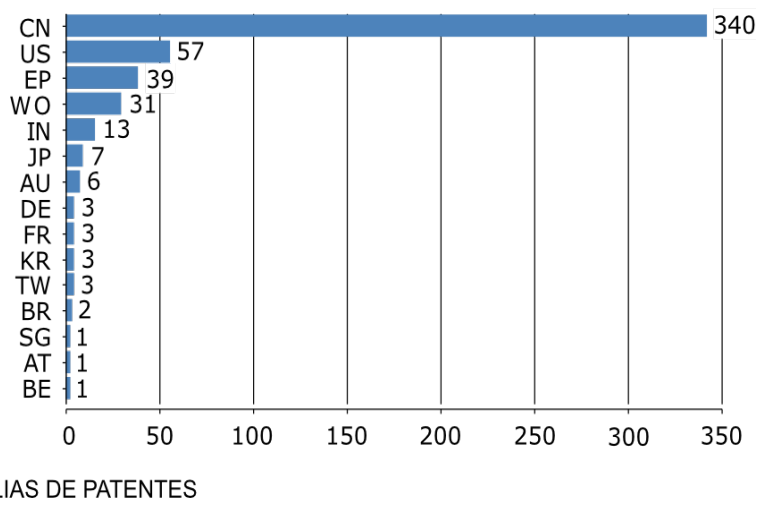

Fonte: Elaborado pelos autores deste artigo a partir da base de dados Orbit Intelligence (2020)

É possível visualizar quem são os depositantes das patentes, e, nesse tópico, as Estratégias de Buscas I e II apresentam os mesmos resultados. Como mostra o Gráfico 4, nota-se que a maioria dos depósitos são de empresas, destaque para as empresas chinesas Shandong Information Technology e Oneconnect Technology nas primeiras posições com maior número de patentes, 14 e cinco, respectivamente. O Bank of America e Wallmart Apollo tem quatro depósitos, porém no quesito classificação alfabética nas bases do Orbit a primeira empresa citada encontra-se na posição cinco, por conseguinte a empresa varejista está na posição onze do ranking, ambas de origem americana.

Da empresa Shandong Information Technology, todas as patentes recuperadas neste trabalho são de métodos de gerenciamento para blockchain e descrevem como será feita a inserção dos dados no bloco de informações em cada etapa do(s) caminho(s). Além disso, o China Blockchain Patents Report 2019 considerou a Oneconnect como a segunda colocada em patentes expressivas, o ranking leva em consideração fatores como o número de pedidos de patente, o número de patentes concedidas, o escopo da proteção de patentes, a localização da família de 
patentes e o pedido de patente. Esse resultado é validado no gráfico apresentado a seguir, e a empresa encontra-se na segunda posição nos resultados dos escopos deste estudo. Além disso, a Oneconnect possui a plataforma blockchain, FiMAX, que, entre as soluções de aplicações, estão fintechs, cadeia de suprimentos, proteção ambiental inteligente, cidade inteligente e métodos de gerenciamento (PRNEWSWIRE, 2019).

O Bank of America é um dos maiores bancos americanos e tem realizado vários depósitos com blockchain (82 patentes) para soluções de financiamento comercial, apesar da sua relação com setor financeiro, essa empresa tem apostado também na tecnologia com uso para cadeias de suprimentos. No entanto, entre as três patentes recuperadas, apenas uma foi concedida, que data de 2019, e reivindica método para gerenciamento de itens de ordem física para rastreio desde a fabricação, a remessa até a venda feita por meio de um ou mais sensores ligados a softwares de terceiros, que, por sua vez, se interligam a blockchain da empresa (BANK OF AMERICA, 2019).

Vale destacar que a Walmart Apollo, uma das principais varejistas americana, que nos últimos anos tem buscado ativamente vários métodos de aplicações blockchain para sua cadeia de suprimento de alimentos, é apontada como uma das líderes em depósitos no ocidente. No Gráfico 4, ela aparece na $11^{\mathrm{a}}$ posição em número de depósitos. Outros registros do Walmart incluem sistema "Smart Package" para rastrear o conteúdo da embalagem, condições ambientais, localização e outros detalhes, e uma solução elétrica que será alimentada por Bitcoin ou outras criptomoedas (ALEXANDRE, 2018).

Das quatro patentes da Walmart, apenas uma foi concedida, data de 2019, e reivindica uma metodologia para aplicação em rótulos inteligentes com informações de temperatura em produtos da cadeia de frios, os produtos passam informações ao pacote por meio de radiofrequência (RFID), que, por sua vez, se interliga ao blockchain armazenado em um sistema de computador preexistente. Com isso, em qualquer etapa do transporte poderá ocorrer troca de informações dos dados, que podem ser analisados novamente com o propósito de gerar a segurança da cadeia (WALLMART APOLLO, 2019).

Gráfico 4 - Número de patentes relacionado aos depositantes

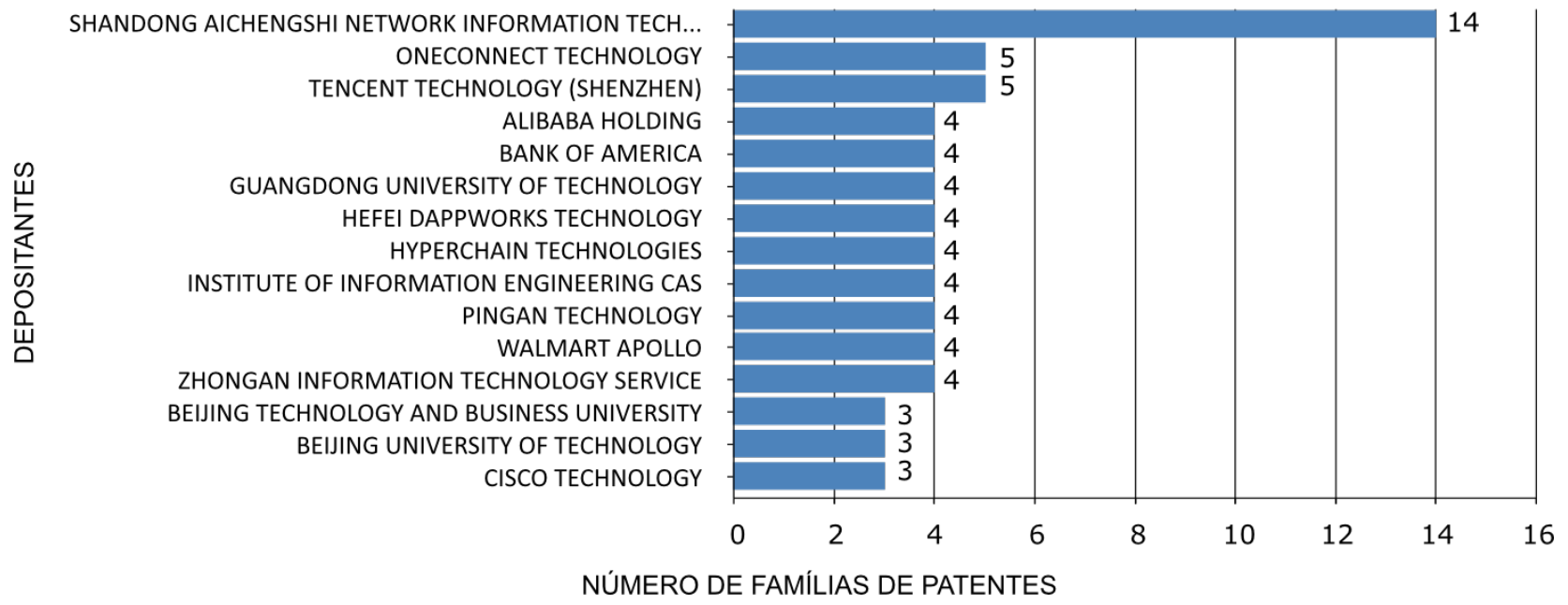

Fonte: Elaborado pelos autores deste artigo a partir da base de dados Orbit Intelligence (2020) 
Diante das informações de 411 patentes da Estratégia de Busca I, no Gráfico 5, serão apontados os depósitos que se destacam por meio dos Domínios Tecnológicos (Technology Domains), que são grupos derivados dos códigos CIPs, sua utilização torna possível melhorar a visualização das áreas das publicações das patentes. Os três domínios mais utilizados são para fins de Métodos de Gerenciamento (It Methods For Management) com 56,61\%, seguidos de Comunicação Digital (Digital Communication) com 24,64\% e Tecnologias Computacionais (Computer Technology) com 13,39\%. No item outros (Others - 5,36\%) estão inseridos domínios que remetem: a Controle (Control), à Medição (Measurement), a Telecomunicações (Telecommunications), a Máquinas Elétricas (Electrical Machinery), Games, à Manipulação (Handling) e à Análise de Materiais Biológicos (Analysis of Biological Materials).

Gráfico 5 - Relação de Domínios Tecnológicos a partir dos resultados das publicações

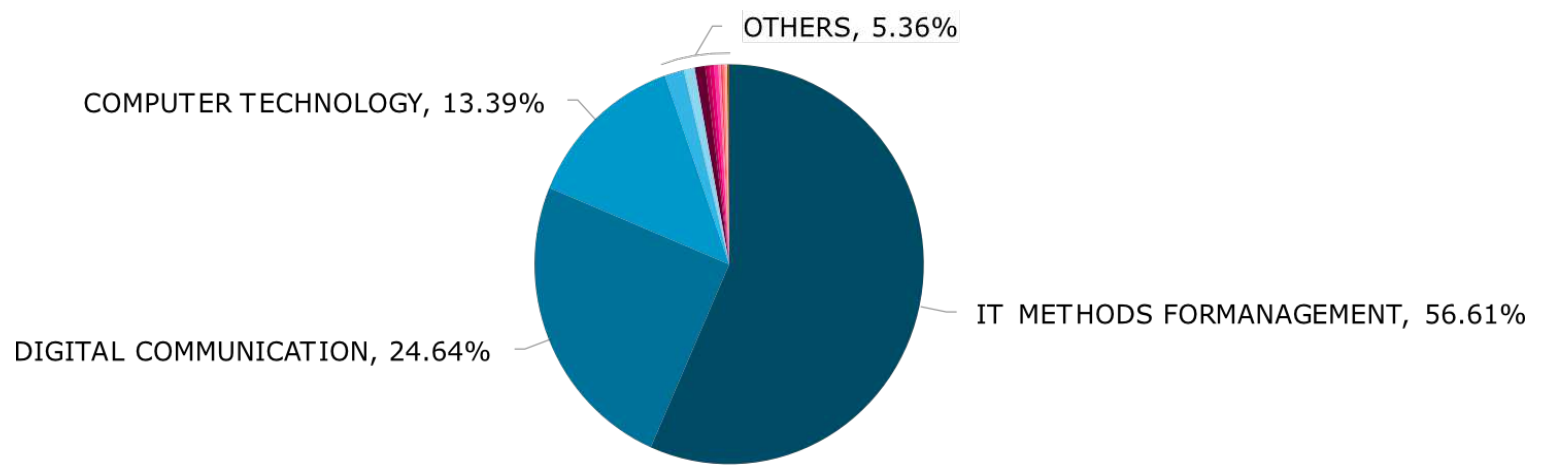

Fonte: Elaborado pelos autores deste artigo a partir da base de dados Orbit Intelligence (2020)

Da mesma forma, com o uso da Estratégia de Busca II e das 423 famílias de patentes recuperadas, os mais reivindicados são os de Métodos de Gerenciamento com 55,67\%, seguidos de Comunicação Digital com 25,26\% e Tecnologias Computacionais com 13,23\%. Nesse caso, outros (Others $-5.84 \%$ ) domínios que aparecem neste resultado, diferente do gráfico anterior, são de Outras Máquinas Especiais (Other Special Machines), Tecnologias Audiovisual (Audio-Visual Technology), Química de Materiais Básico (Basic Materials Chemistry) e Tecnologia médica (Medical Technology). Apesar de ser perceptível a diferença mínima quanto ao escopo 1 de busca, nota-se um acréscimo no item Comunicação Digital, partindo-se da ideia de novos dispositivos, hardwares para comunicação na utilização em cadeias de suprimentos e blockchain, enquanto se vê leve baixa no domínio de Métodos de Gerenciamento.

Ainda sobre patentes, o Brasil em sua Lei de Software (Lei n. 9.609/98) não aceita patentes para software por conferir a estes as obras literárias (Lei n. 9.610/98), ou seja, o país reconhece apenas seu registro. Em vista disso, foi realizada uma busca na base de dados do INPI, seção Programa de Computador, na tentativa de encontrar registros referentes à temática da pesquisa com uso do campo "Título do programa" apenas com a palavra-chave "Blockchain", e foram recuperados 16 registros, mas apenas um se correlaciona ao assunto deste trabalho. Tal registro tem como titular o Centro de Pesquisa e Desenvolvimento em Telecomunicações (CPQD), cujo título do registro é Rastreando a Cadeia de Carne Brasileira com Blockchain, publicado na Revista da Propriedade Industrial, edição 2.556.

Isso sugere que, apesar de o Brasil ter baixa participação em criação de número de patentes (apenas um apontado no Gráfico 3) e um registro de software, o país começa a enxergar como viés mercadológico a tecnologia blockchain para uso em cadeias de suprimentos. 


\subsection{Publicações Científicas}

Os números alcançados com as buscas servem para visualização temporal das publicações científicas encontradas sobre o tema, e, com refinamento dos operadores, foi possível obter o resultado de 1.303 documentos que decorrem deste estudo de forma mais completa, abrangendo todas as palavras-chave dentro do contexto que se buscou. No Gráfico 6(A), os dados de publicações científicas recuperados quanto ao ano de publicação versus o quantitativo de documentos mostram correlação com o Gráfico 2 de patentes, em que se tem, em 2018 e 2019, o auge de quantidade de publicações, tanto de patentes quanto de produções científicas. No ano de 2020, é visível a queda no resultado de publicações devido ao período de busca deste trabalho datar-se até junho deste ano, portanto, a tendência é que o número aumente no decorrer do ano.

É possível observar que novamente a China desponta como o país com a maior quantidade de publicações sobre a temática, Gráfico 6(B), total de 293 documentos científicos. Em seguida, se destacam Estados Unidos e Índia na segunda e terceira posição, respectivamente. O Brasil aparece na $23^{\mathrm{a}}$ posição no ranking, tendo publicado 17 documentos científicos. É importante observar que, diferente de patentes, a China no quesito publicações científicas não desponta na frente, mostra-se também além dos Estados Unidos, Índia, Reino Unido, Alemanha e Austrália, países que têm investido em pesquisas para aplicações da tecnologia blockchain.

O documento mais bem referenciado foi publicado em uma conferência em 2016 na China e contextualiza os problemas de segurança alimentar do país. O artigo aborda a utilização e o desenvolvimento da tecnologia RFID (Radio-Frequency IDentification) e blockchain, ainda analisa as vantagens e as desvantagens do uso da tecnologia RFID e blockchain na construção do sistema de rastreabilidade da cadeia de suprimentos agroalimentar, e, como parte dos resultados, demonstra o processo de construção desse sistema (FENG, 2016).

Pelos Estados Unidos, o segundo documento mais bem posicionado, é afiliado pela University of North Carolina com o título de Can Blockchain Strengthen the Internet of Things? O documento avalia como tecnologia blockchain pode fortalecer a segurança na Internet das Coisas (IoT), já que a blockchain se mostra superior ao ecossistema de IoT por ter servidores em nuvem centralizados, a blockchain funciona de forma descentralizada (KSHETRI, 2017).

Gráfico 6 - (A) Número de publicações por ano; (B) Países com mais publicações científicas

A

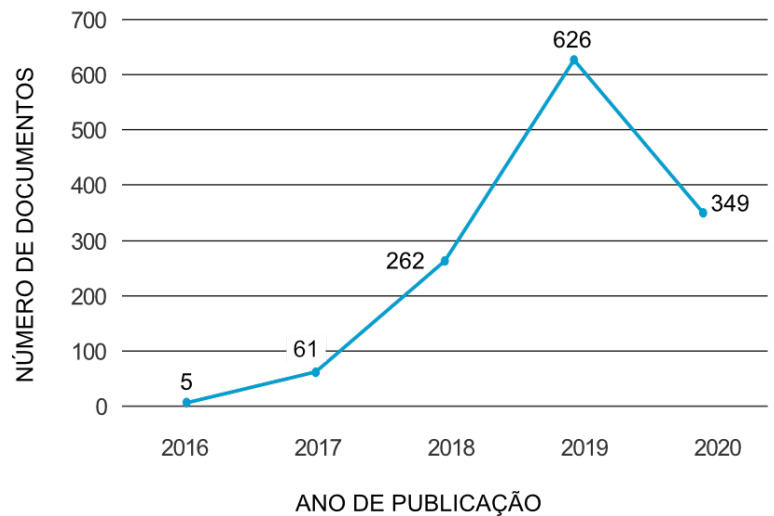

B

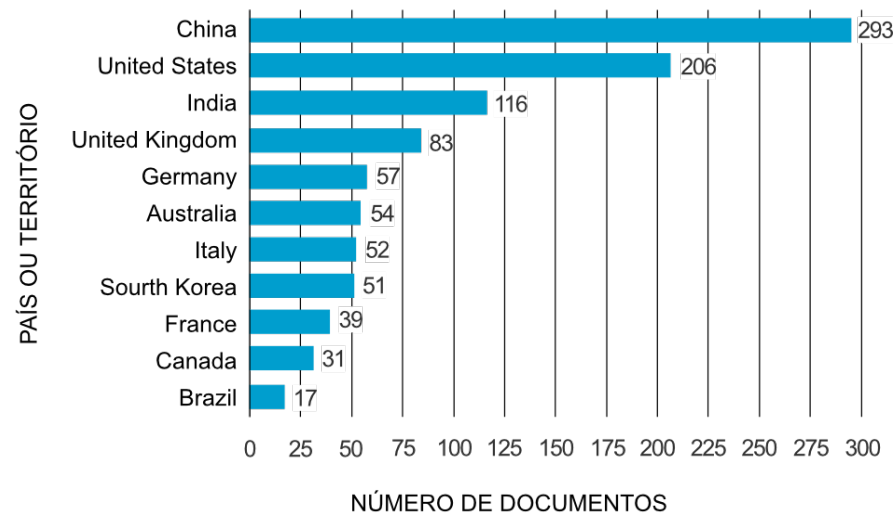


Fonte: Elaborado e adaptado pelos autores deste artigo a partir da base de dados Scopus (2020)

Das 1.303 publicações científicas, destacam-se as publicações do pesquisador Khaled Salah, da Khalifa University of Science and Technology, de Abu Dhabi, nos Emirados Árabes, ele é coautor de nove documentos amplamente referenciados na literatura. A partir do pressuposto de que os Emirados Árabes Unidos é que mais publica na região do Oriente Médio, em pesquisas complementares, destaca-se que o governo possui estratégia para tornar Abu Dhabi até o final de 2021 uma cidade que funcionará toda baseada em blockchain (BASHIR; ALFAHAM, 2018). O primeiro e o segundo autor, ver Gráfico 7(A), possuem trabalhos publicados sobre temática desta pesquisa pela Arizona State University.

Nos Estados Unidos, a Universidade do Arizona está entre os cinco programas de pós-graduação para estudos de cadeias de suprimentos com uso de blockchain mais conceituados do país (U.S. NEWS \& WORLD REPORT, 2020). O estado do Arizona, entre outros estados americanos, aposta fortemente na tecnologia blockchain, uma vez que aprovou em 2018 uma lei que permitiu a exploração de blockchain nos setores de finanças, smart contracts, e regulamenta corporações a manterem e a compartilharem dados na blockchain para acesso livre (HUILLET, 2018).

Outro destaque é de um pesquisador brasileiro tendo coautoria em cinco documentos científicos, sendo um destes utilizado como base para outros trabalhos, pois traz uma revisão sistemática da integração do blockchain às cadeias de suprimentos (QUEIROZ; TELLES; BONILLA, 2019).

Gráfico 7 - (A) Autores de publicações científicas; (B) Produções científicas por Instituição

A

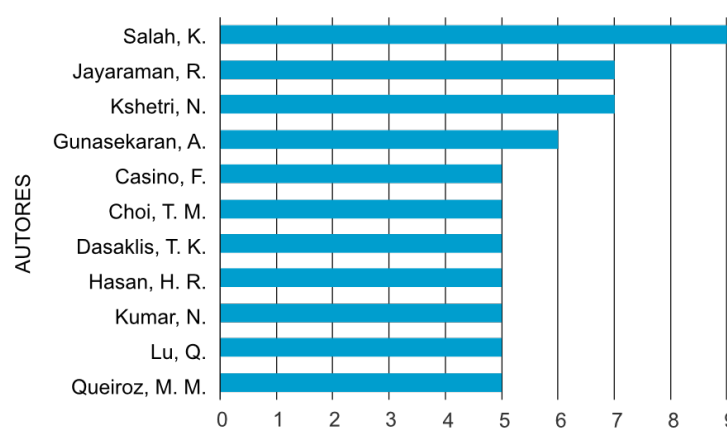

B

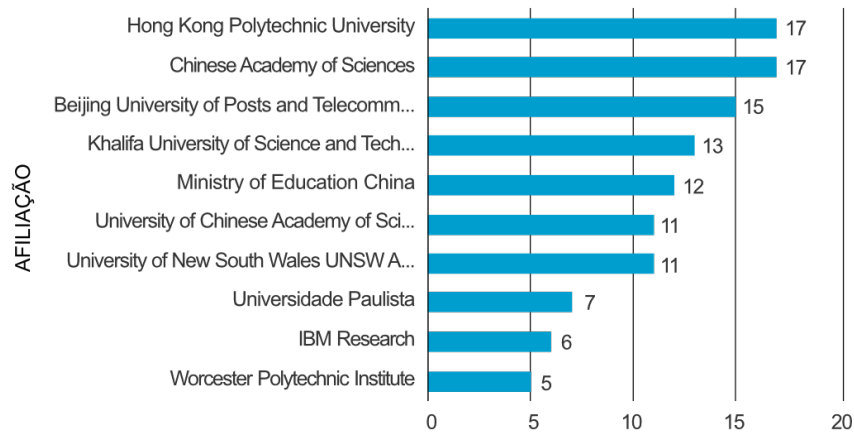

NÚMERO DE DOCUMENTOS

Fonte: Elaborado pelos autores deste artigo a partir de dados da base Scopus (2020)

Dentro do escopo científico, as instituições de ensino assumem o papel de auxiliadoras do meio, seja qual for o caminho a seguir de uma tecnologia relativamente nova. O Gráfico 7(B) destaca as instituições que têm se sobressaído na temática abordada, que é a principal fomentadora de conhecimento com 11 documentos científicos. A Hong Kong Polytechnic University em 2018 criou em conjunto com parceiros australianos um laboratório para estudos avançados focado em tecnologias blockchain, sendo referência nos estudos das várias aplicações da tecnologia (PRNEWSWIRE, 2018). 
Vale adendo para a Universidade Paulista que possui sete artigos publicados que fazem referências conceituais e investigativa de cenários de aplicação de blockchain. Não foi encontrada relação de estratégia de incentivo de pesquisa em instituição específica para a tecnologia.

A empresa de tecnologia IBM possui um laboratório de pesquisas para tecnologias inovadores, o IBM Research. Esse player encontra-se entre os mais expressivos no mundo quanto ao assunto de blockchain e tem se mostrado estar à frente em várias ações que frisam o rastreio de cadeias de suprimentos, como já mencionado, com uso da ferramenta Food Trust (IBM, 2020). Neste estudo, foram encontradas seis contribuições científicas advindas do centro de pesquisa da empresa.

A Worcester Polytechnic Institute, em Massachusetts, Estados Unidos, possui um dos cinco artigos mais bem posicionados e referenciados por outros autores, cita-se aqui o artigo: Blockchain technology and its relationships to sustainable supply chain management (SARKIS et al., 2019). No referido artigo da Worcester, é examinada a aplicação técnica da tecnologia com setores de cadeias de suprimentos que traz ao debate a relação das pressões locais e globais de governo e consumidor quanto às metas de sustentabilidade.

\section{Considerações Finais}

Neste estudo, destacam-se algumas observações e conclusões a respeito da prospecção tecnológica apresentada. Primeiro, no contexto das patentes, demonstrou-se a tecnologia blockchain com potencial para as cadeias de suprimentos, uma vez que sua criação foi voltada para o mercado financeiro por meio das criptomoedas, e isso foi mostrado pelos resultados primários dos códigos CIPs. Indica importantes aspectos da tecnologia como as seções e as subclasses mais solicitadas atualmente para área, os países e os inventores das soluções tecnológicas que se destacam, sendo possível perceber quão promissor é a área devido ao aumento de patentes ao longo dos anos.

O crescente número de patentes e de publicações sobre blockchain em cadeias de suprimentos mostra o potencial que a tecnologia pode ter em um futuro/presente na possibilidade de transformar setores tradicionais. O segundo aspecto, voltado para as publicações científicas, demonstrou o esforço de eficiência, o rastreio e a sustentabilidade diretamente ligados aos documentos científicos, já que, por meio da prospecção, é possível visualizar que as publicações científicas englobam todas as palavras-chave, relacionadas ao tema (blockchain, cadeias de suprimentos e rastreabilidade). Enquanto nas patentes, a palavra-chave "sustentabilidade" não faz relação com nenhum documento recuperado, sendo assim, o domínio tecnológico "métodos de gerenciamento" é o mais aplicado.

Constatou-se no geral que as empresas que se encontram no topo de pesquisas em blockchain estão na seara de proteção de métodos de gerenciamento que demonstra foco em "como fazer" e em "como interagir" com as demais tecnologias. Nesse sentido, observou-se a dependência em outras tecnologias existentes em apoio ao blockchain para as cadeias de suprimentos, como infravermelho, RFID, smart contracts, etiquetas e rótulos inteligentes utilizando QR Codes.

Entre as condicionantes deste trabalho, a busca de anterioridade demonstrou que a China se firma como líder de uso e de pesquisas da tecnologia blockchain com base na relação de quantidade com números de patentes e publicações científicas recuperadas. Neste último que- 
sito (publicações científicas), foi possível visualizar que outros países, como Estados Unidos, Índia e Reino, têm crescido com os estudos na aplicação de blockchain para o tema. A liderança chinesa e americana é reafirmada ao se visualizar que as empresas e os governos desses países possuem estratégias de futuro, de investimentos para pesquisas e de utilização da tecnologia blockchain, tudo identificado pelos dados encontrados por meio dos documentos científicos, além do que, alguns players já se tornam referência para os demais interessados na tecnologia e suas aplicações.

\section{Perspectivas Futuras}

Pela aplicabilidade da tecnologia blockchain é notório que ela poderá ser implementada em variados setores, com mais afinco no setor das cadeias de suprimentos, uma vez que as companhias precisam atender às demandas novas e necessárias, como as de origem de produção e comprovação de sustentabilidade, cada vez mais exigidas pelos consumidores. Porquanto, a maioria das patentes envolve modelos de gerenciamento, abre-se, então, uma perspectiva de que a Internet das Coisas (IoT) e a Inteligência Artificial (IA) assumam importante base de apoio e de conversação entre as mais diversas tecnologias já existentes com as plataformas de blockchain atuais (público, privado, consórcio ou semiprivativo) e as que surgirão.

Aqui se salienta a herança das criptomoedas, os smart contracts, tendo suma importância para segurança e confiabilidade da informação durante todo processo de produção, logística e venda de produtos pela não necessidade de intermediador. Esse elo criado demonstra que um blockchain sem uma infraestrutura de sustentação não passa de conceitos e de utopia.

Considera-se a originalidade deste trabalho as buscas de players globais que utilizam a tecnologia blockchain aplicado ao tema do estudo, sendo possível olhar para o Brasil e notar um modesto destaque no cenário mundial, visto que nenhuma aplicação dessa tecnologia direciona-se para a proteção da Amazônia, mesmo com latente cenário de busca da preservação ambiental. Logo, seriam ideais ações e mecanismos de rastreio com blockchain, a fim de se criar a transparência dos dados, tanto de atos do país quanto das ações de empresas que exploram recursos naturais da floresta, pois falta uma "política de Estado" que incentive e financie estudos para essa tecnologia, ou mesmo cooperação para uso dentro do estado brasileiro.

Finalmente, diante da visão apresentada, este trabalho será expandido com o aprofundamento nas pesquisas de classificações, uma vez que, para o tema e suas peculiaridades, as subclasses CIP ainda não possuem indicações assertivas, próprias. Como já se mencionou, a construção do roadmap tecnológico surge como uma solução, já que contribui para as inter-relações de tudo que foi encontrado nas etapas anteriores e para a realimentação dos resultados obtidos neste estudo.

\section{Referências}

ABADI, M.; BALL, A. Palm oil plantations are under pressure to prove they're sustainable: but the process can have devastating effects on local communities and the plantations themselves. Publicado em maio de 2020. Disponível em: https://www.businessinsider.com/palm-oil-plantationsustainable-environment-guatemala-2020-4. Acesso em: 2 jun. 2020. 
ABRAPALMA - ASSOCIAÇÃO BRASILEIRA DE PRODUTORES DE ÓLEO DE PALMA. Retrospecto e projeções da palma de óleo no Brasil 2018-2019. Dezembro de 2018. Disponível em: http://www.abrapalma.org/pt/wp-content/uploads/2018/12/Resumo_ Relatorio_2018-2.pdf. Acesso em: 2 jul. 2020.

ALEXANDRE, Ana. Recent Walmart Patent Application Describes Blockchain-Managed Smart Appliances, Aug. 2018. Disponível em: https://cointelegraph.com.br/news/recent-walmartpatent-application-describes-blockchain-managed-smart-appliances. Acesso em: 2 jun. 2020.

AN, Wensen. Patenting blockchain technology in Asia: trends and patentability, Dez. 2019. Disponível em: https://www.lushenglawyers.com/awards-press/patenting-blockchain-technology-inasia-trends-and-patentability/. Acesso em: 2 nov. 2020.

ANZALONE, Robert. IBM Blockchain Is Growing In The Food Industry During Covid-19, 2020. Disponivel em: https://www.forbes.com/sites/robertanzalone/2020/06/04/ibm-blockchaintechnology-is-growing-in-the-food-industry-during-covid-19/?_lrsc=b61ca797-e00b-4255-b515d2aa60bd7a56\#357416007f07. Acesso em: 2 abr. 2020.

BANK OF AMERICA. Blockchain-based property management. Depositante: Bank of America Corporation. US 10,498,808 B2. Depósito: 28 Mar. 2018. Concessão: 3 Dez. 2019.

BASHIR, H.; ALFAHAM, T. Mohammed bin Rashid launches 'UAE Blockchain Strategy'. Publicado em Abril de 2018. Disponível em: http://wam.ae/en/details/1395302681330. Acesso em: 2 jun. 2020.

BORSCHIVER, S.; SILVA, A. L. R. Technology Roadmap: Planejamento Estratégico para alinhar Mercado-Produto-Tecnologia. 1. ed. Rio de Janeiro: Interciência, 2016. ISBN: 9788571933866.

BULL. System and method for the creation and management of decentralized authorizations for connected objects. Depositante: BULL [FR]. Depósito: 22 Dez. 2017. Data da Publicação Nacional: 2 Jan. 2019.

BYRNE, J. Norwegian Company suspends purchase of feed from Cargill, Jul. 2020. Disponível em: https:/www.feednavigator.com/Article/2020/07/07/Grieg-Seafood-pressures-Cargill-overdeforestation-links. Acesso em: 2 jul. 2020.

CANT, J. China torna mais simples processo para pedido de patente blockchain, Jan. 2020. Disponível em: https://cointelegraph.com.br/news/china-continues-to-streamline-its-blockchainpatent-application-process. Acesso em: 2 nov. 2020.

ELSEVIER. Elsevier - Scopus, 2020. Disponível em: https://www.elsevier.com/solutions/scopus. Acesso em: 2 abr. 2020.

FENG, Tian. An agri-food supply chain traceability system for China based on RFID \& blockchain technology, 2016. In: 13TH INTERNATIONAL CONFERENCE ON SERVICE SYSTEMS AND SERVICE MANAGEMENT (ICSSSM), Kunming, 2016, p. 1-6. Anais [...]. Kunming, 2016. DOI: 10.1109/ICSSSM.2016.7538424.

HARTMAN GROUP, National Syndicated Research: Sustainability 2017. [2017]. Disponível em: http://store.hartman-group.com/content/Sustainability-2017-Overview.pdf. Acesso em: 2 abr. 2020.

HE, Laura. Walmart China accused of using old meat, oil, Aug. 2014. Disponível em: https:// www.marketwatch.com/story/walmart-china-accused-of-using-old-meat-oil-2014-08-08?siteid=nbkh. Acesso em: 2 abr. 2020. 
HUILLET, M. Arizona Blockchain Bill Signed Into State Law. [2018]. Disponível em: https:// cointelegraph.com/news/arizona-blockchain-bill-signed-into-state-law. Acesso em: 2 jul. 2020.

IBM. Focus On Sustainability. IBM Food Trust: 2018. Disponível em: https://www.ibm.com/ downloads/cas/R8VDMJ4Y. Acesso em: 2 dez. 2019.

IBM. Research. Science for Society, 2020. Disponível em: https://www.research.ibm.co. Acesso em: 2 jul. 2020.

KSHETRI, N. Can Blockchain Strengthen the Internet of Things? IT Professional, [s.l.], v. 19, n. 4, p. 68-72, 2017. DOI: 10.1109/MITP.2017.3051335.

LESSAK, A. K.; DIAS, R. A.; FREY, I. A. Blockchain: Prospecção Tecnológica em Bases de Patentes. Cadernos de Prospecção, Salvador, v. 11, n. 3, p. 876-887, setembro, 2018.

NAÇÕES UNIDAS. Agenda 2030. [2015]. Disponível em: https://nacoesunidas.org/pos2015/ agenda2030/. Acesso em: 2 jun. 2020.

ORBIT INTELIGENCE. [Ferramenta de busca de bases de dados - Internet]. CQuestel; 2020. Disponível em: www.orbit.com. Acesso em: 3 jul. 2020.

PETERSON, H. Wal-Mart China Accused Of Firing Workers Who Claimed The Company Was Selling Expired Food, Set. 2014. Disponível em: https://www.businessinsider.com/wal-mart-chinafires-workers-2014-9\#ixzz3DnNdTyB. Acesso em: 2 abr. 2020.

PRNEWSWIRE. Alibaba and OneConnect take the top two positions in Blockchain Patents list and file IPO prospectuses on the same day. Publicado em Nov. 2019. Disponível em: https:// www.prnewswire.com/news-releases/alibaba-and-oneconnect-take-the-top-two-positions-in-blockchainpatents-list-and-file-ipo-prospectuses-on-the-same-day-300965098.html. Acesso em: 2 jul. 2020.

\section{PRNEWSWIRE. PolyU Joins Forces with Australian Partners in Launching Impactful}

Research on Blockchain Technologies. Publicado em Mar. 2018. Disponível em: https://www. prnewswire.com/news-releases/polyu-joins-forces-with-australian-partners-in-launching-impactfulresearch-on-blockchain-technologies-677940293.html. Acesso em: 2 jul. 2020.

QUEIROZ, M. M.; TELLES, R.; BONILLA, S. H. Blockchain and supply chain management integration: a systematic review of the literature. Supply Chain Management, [s.l.], v. 25, n. 2, p. 241-254, 2019. DOI: https://doi.org/10.1108/SCM-03-2018-0143.

QUINTELLA, Cristina M. et al. Prospecção Tecnológica como uma Ferramenta Aplicada em Ciência e Tecnologia para se Chegar à Inovação. Revista Virtual de Química, [s.l.], v. 3, n. 5, p. 406-415, dez. 2011. Disponível em: http://rvq-sub.sbq.org.br/index.php/rvq/article/view/193. Acesso em: 2 abr. 2020.

RAMANURTHY, Shanker. Leveraging blockchain to improve food supply chain traceability. [2016]. Disponível em: https://www.ibm.com/blogs/blockchain/2016/11/leveraging-blockchainimprove-food-supply-chain-traceability/. Acesso em: 2 dez. 2019.

RAPOSO, Marco T. O. et al. Equipamento, sistema e processo de rastreabilidade, monitoramento e certificação da cadeia do leite e derivados. Depositante: Marco Tulio de Oliveira Raposo. BR 102018069615-7 A2. Depósito: 25 Set. 2018. Data da Publicação Nacional: 7 Abr. 2020.

REVISTA DA PROPRIEDADE INDUSTRIAL, [s.l.], n. 2.556, publicada em dezembro de 2019. Disponível em: http://revistas.inpi.gov.br/rpi/. Acesso em: 2 jul. 2020. 
RYAN, M. Ethics of Using AI and Big Data in Agriculture: the Case of a Large Agriculture Multinational. ORBIT Journal, [s.l.], v. 2, n. 2, 2019. https://doi.org/10.29297/orbit.v2i2.109

SARKIS, Joseph et al. Blockchain technology and its relationships to sustainable supply chain management. International Journal of Production Research, [s.l.], v. 57, n. 7, p. 2.117-2.135, 2019. DOI: $10.1080 / 00207543.2018 .1533261$.

SMART, Press. UAE Towards Its Goal to Become a Fully Blockchain-powered City By 2021, Publicado em Fev. 2020. Disponível em: https://www.smartcity.press/uae-blockchain-strategy/. Acesso em: 2 jun. 2020.

\title{
U.S. NEWS \& WORLD REPORT. Best Undergraduate Supply Chain Management/Logistics
}

Programs, 2020. Disponível em: https://www.usnews.com/best-colleges/rankings/business-supplychain-management-logistics. Acesso em: 2 jun. 2020.

WALLMART APOLLO. Blockchain-based system and method for supply chain control. Depositante: Walmart Apollo, LLC. US 10,521,806 B2. Depósito: 25 Fev. 2019. Concessão: 31 Dez. 2019.

WORLD ECONOMIC FORUM. Strategic Intelligence - Global Issue: Blockchain. Disponível em: https://intelligence.weforum.org. Acesso em: 2 abr. 2020.

\section{WIPO - WORLD INTELLECTUAL PROPERTY ORGANIZATION. Internacional Patent}

Classification (IPC). 2020. Disponível em: https://www.wipo.int/classifications/ipc/en/ Acesso em: 2 Jan. 2020.

\section{Sobre os Autores}

\author{
Andrik Guimarães Ferreira \\ E-mail: andrikferreira@outlook.com \\ ORCID: http://orcid.org/0000-0003-4306-3047 \\ Pós-Graduado Lato Sensu pelo Instituto Esperança de Ensino Superior, Informática na Educação em 2016. \\ Endereço profissional: Instituto Esperança de Ensino Superior, Rua Deputado Icoaraci Nunes Caranazal, Santarém, \\ PA. CEP: 68035-110.
}

\section{Rosinei de Sousa Oliveira}

E-mail:nei.ufopa@gmail.com

ORCID: http://orcid.org/0000-0002-2427-5020

Pós-Doutor pelo Instituto de Desenvolvimento de Novas Tecnologias, UNINOVA, Portugal.

Ciências Sociais Aplicadas em 2019.

Endereço profissional: Universidade Federal do Oeste do Pará, Instituto de Engenharia e Geociências, Rua Vera Paz Salé, Santarém, PA. CEP: 68035-110.

\section{Cristina M. Quintella}

E-mail: cris5000tina@gmail.com

ORCID: http://orcid.org/0000-0002-3827-7625

Pós-Doutora pelo Instituto Superior de Economia e Gestão da Universidade de Lisboa, ISEG, Portugal. Ciências Sociais Aplicadas em 2020.

Endereço profissional: Universidade Federal da Bahia, Instituto de Química, Departamento de Química Geral e Inorgânica, Campus de Ondina, Ondina, Salvador, BA. CEP: 40170-290. 


\section{Celson Pantoja Lima}

E-mail: celson.pantoja@gmail.com

ORCID: http://orcid.org/0000-0002-8074-8566

Pós-Doutor pelo Massachusetts Institute of Technology, MIT, Estados Unidos.

Engenharias em 2015.

Endereço profissional: Universidade Federal do Oeste do Pará, Instituto de Engenharia e Geociências, R. Vera Paz, s/n (Campus Tapajós), Salé, Santarém, PA. CEP: 68035-110. 